\title{
MINIMAL SURFACES IN A UNIT SPHERE PINCHED BY INTRINSIC CURVATURE AND NORMAL CURVATURE
}

\author{
YANG DAN
}

\begin{abstract}
We establish a nice orthonormal frame field on a closed surface minimally immersed in a unit sphere $S^{n}$, under which the shape operators take very simple forms. Using this frame field, we obtain an interesting property $K+K^{N}=1$ for the Gauss curvature $K$ and the normal curvature $K^{N}$ if the Gauss curvature is positive. Moreover, using this property we obtain the pinching on the intrinsic curvature and normal curvature, the pinching on the normal curvature, respectively.
\end{abstract}

\section{INTRODUCTION}

Let $x: M \rightarrow S^{n}$ be a minimal immersion of a closed surface $M$ into an ndimensional unit sphere $S^{n}$. Let $K_{s}=2 /(s(s+1))$ for each natural number $s$. Using an idea of Hopf and the global coordinates on $S^{n}$, Calabi [3] proved that if $M$ is a 2-sphere with constant Gauss curvature $K_{s}$ and and $x$ is linearly full, then $n=2 s$ and $x$ is congruent to $s$-th standard minimal immersion. For more general minimal immersion of 2-sphere into $S^{n}$, Chern [4, p. 38] obtained an important equality about some local invariants by choosing a local orthonormal frame filed on $S^{n}$. As a special case, Chern showed that if the Gauss curvature $K$ is constant then $K=K_{s}$. Furthermore, for general minimal immersion of a surface into $S^{n}$, Kenmotsu 9, 10, also obtained an important equality (see Theorem 1 in 9]) by choosing the frame filed, which generalized Chern's result for the minimal immersion of 2-sphere into $S^{n}$. We could observe that the chosen of the frame filed plays an important role in studying the minimal surface. The first purpose of this paper is to establish a best local orthonormal frame field on the closed surface minimally immersed in $S^{n}$ with positive Gauss curvature, under which the shape operators take the most simple forms, see Theorem 2.3 for detail.

Furthermore, using the frame field introduced in Theorem[2.3, we can obtain an interesting result $K+K^{N}=1$ for the Gauss curvature $K$ and the normal curvature $K^{N}$, which means that closed minimal surfaces immersed in $S^{n}$ with positive Gauss curvature and flat or nowhere flat normal bundle are Wintgen ideal surfaces, see Theorem 3.3. Wintgen ideal submanifolds are a family of submanifolds satisfying a DDVV type inequality when the equality holds true exactly, see [5] for instance.

Recently, a remarkable result due to Baker and Nguyen [1] says that codimensional two surfaces satisfying a nonlinear curvature condition depending on normal curvature smoothly evolve by mean curvature flow to round points. In the course of estimating the nonlinearity in the Simons identity, the authors announced an

2000 Mathematics Subject Classification. Primary 53C42; Secondary 53A10.

Key words and phrases. Minimal surface; normal curvature; Gauss curvature; pinching. 
interesting result depending on a pointwise pinching of the intrinsic and normal curvatures.

Theorem 1.1. ( [1) Suppose a two surface $M$ minimally immersed in $\mathbb{S}^{4}$ satisfies $K^{N} \leq 2|K|$. Then either

(1) $S=0$ and $M$ is a geodesic sphere; or

(2) $S \neq 0$, in which case either

(a) $K^{N}=0$ and the surface is the Clifford torus, or

(b) $K^{N} \neq 0$ and it is the Veronese surface.

By using the frame field obtained in Theorem 2.3. we generalize this result to the minimal surfaces in arbitrary dimension unit sphere $S^{n}$. We prove that closed minimal surfaces immersed in $S^{n}$ with nonnegative Gauss curvature and flat or nowhere flat normal bundle satisfying $K^{N} \leq 2 K$ are geodesic sphere, the Clifford torus, or the Veronese surface in $S^{4}$, see Theorem 3.6 for detail.

Based on this result, we continue to consider the next pinching $2 K \leq K^{N} \leq$ $5 K$, see Theorem 3.8. Then we study the first pinching of normal curvature $0 \leq$ $K^{N} \leq 2 / 3$, see Theorem 3.9, and the next pinching $2 / 3 \leq K^{N} \leq 5 / 6$, see Theorem 3.10. At last, we prove that closed surfaces minimally immersed in $S^{n}$ with positive Gauss curvature and non-zero constant normal curvature are generalized Veronese surfaces studied by Calabi [3] and do-Carmo-Wallach [6].

The paper is organized as follows. In Section 2, we introduce some basic formulae for theory of submanifolds and establish an orthonormal frame field on the closed surfaces minimally immersed in a unit sphere, which is crucial to get the main theorems. In Section 3, we give some pinching theorems and their proofs.

\section{Basic formulae and the frame field}

Let $M$ be a closed surface immersed in a unit sphere $S^{n}$. We identify $M$ with its immersed image, agree on the following index ranges:

$$
1 \leq i, j, k, l, m, \cdots \leq 2 ; \quad 3 \leq \alpha, \beta, \gamma, \delta, \cdots \leq n ; \quad 1 \leq A, B, C, D, \cdots \leq n,
$$

and use the Einstein convention. We take a local orthonormal frame field $\left\{e_{1}, \cdots, e_{n}\right\}$ in $T S^{n}$ such that, restricted to $M$, at each point of $M,\left\{e_{1}, e_{2}\right\}$ lies in the tangent bundle $T(M)$ and $\left\{e_{3}, \cdots, e_{n}\right\}$ in the normal bundle $N(M)$. Let $\left\{\omega_{1}, \cdots, \omega_{n}\right\}$ be the dual coframe field of $\left\{e_{1}, \cdots, e_{n}\right\}$ and $\left(\omega_{A B}\right)$ the Riemannian connection form matrix associated with $\left\{\omega_{1}, \cdots, \omega_{n}\right\}$. Then $\left(\omega_{i j}\right)$ defines a Riemannian connection in $T(M)$ and $\left(\omega_{\alpha \beta}\right)$ defines a normal connection in $N(M)$. The second fundamental form of $M$ can be expressed as

$$
I I=\omega_{i} \otimes \omega_{i \alpha} \otimes e_{\alpha}=h_{i j}^{\alpha} \omega_{i} \otimes \omega_{j} \otimes e_{\alpha}
$$

where

$$
\omega_{i \alpha}=h_{i j}^{\alpha} \omega_{j} ; \quad h_{i j}^{\alpha}=h_{j i}^{\alpha} .
$$

Let $L^{\alpha}=\left(h_{i j}^{\alpha}\right)_{2 \times 2}$. We denote the square of the norm of the second fundamental form $S$ by

$$
S=\sum_{(\alpha, i, j)}\left(h_{i j}^{\alpha}\right)^{2} .
$$

The mean curvature vector field of $M$ is expressed as

$$
h=\frac{1}{2} \sum_{\alpha=3}^{n}\left(h_{11}^{\alpha}+h_{22}^{\alpha}\right) e_{\alpha},
$$


then $M$ is minimal if and only if $h=0$. The Riemannian curvature tensor $\left\{R_{i j k l}\right\}$ and the normal curvature tensor $\left\{R_{\alpha \beta k l}\right\}$ are expressed as

$$
R_{i j k l}=\left(\delta_{i k} \delta_{j l}-\delta_{i l} \delta_{j k}\right)+h_{i k}^{\alpha} h_{j l}^{\alpha}-h_{i l}^{\alpha} h_{j k}^{\alpha}, \quad R_{\alpha \beta k l}=h_{k m}^{\alpha} h_{m l}^{\beta}-h_{l m}^{\alpha} h_{m k}^{\beta} .
$$

We denote the normal scalar curvature $K^{N}$ by

$$
K^{N}=\frac{1}{2} \sqrt{\sum_{(\alpha, \beta, i, j)}\left(R_{\alpha \beta i j}\right)^{2}} .
$$

The first and the second order covariant derivatives of $\left\{h_{i j}^{\alpha}\right\}$, say $\left\{h_{i j k}^{\alpha}\right\}$ and $\left\{h_{i j k l}^{\alpha}\right\}$ are defined as follows:

$$
\begin{gathered}
\nabla h_{i j}^{\alpha}=h_{i j k}^{\alpha} \omega_{k}=d h_{i j}^{\alpha}+h_{m j}^{\alpha} \omega_{m i}+h_{i m}^{\alpha} \omega_{m j}+h_{i j}^{\beta} \omega_{\beta \alpha}, \\
\nabla h_{i j k}^{\alpha}=h_{i j k l}^{\alpha} \omega_{l}=d h_{i j k}^{\alpha}+h_{m j k}^{\alpha} \omega_{m i}+h_{i m k}^{\alpha} \omega_{m j}+h_{i j m}^{\alpha} \omega_{m k}+h_{i j k}^{\beta} \omega_{\beta \alpha} .
\end{gathered}
$$

Then we have the Codazzi equation

$$
h_{i j k}^{\alpha}=h_{i k j}^{\alpha}
$$

and the Ricci's formula

$$
h_{i j k l}^{\alpha}-h_{i j l k}^{\alpha}=h_{p j}^{\alpha} R_{p i k l}+h_{i p}^{\alpha} R_{p j k l}+h_{i j}^{\beta} R_{\beta \alpha k l} .
$$

The Laplacian of $\left\{h_{i j}^{\alpha}\right\}$ and $\left\{h_{i j k}^{\alpha}\right\}$ are defined by

$$
\Delta h_{i j}^{\alpha}=h_{i j m m}^{\alpha}, \quad \Delta h_{i j k}^{\alpha}=h_{i j k m m}^{\alpha} .
$$

It follows from (2.2) and (2.3) that

$$
\begin{aligned}
\Delta h_{i j}^{\alpha} & =h_{m m i j}^{\alpha}+h_{p i}^{\alpha} R_{p m j m}+h_{m p}^{\alpha} R_{p i j m}+h_{m i}^{\delta} R_{\delta \alpha j m} \\
\Delta h_{i j k}^{\alpha} & =\left(\Delta h_{i j}^{\alpha}\right)_{k}+2 h_{p j m}^{\alpha} R_{p i k m}+2 h_{i p m}^{\alpha} R_{p j k m}+h_{i j p}^{\alpha} R_{p m k m} \\
& +h_{p j}^{\alpha} R_{p i k m, m}+h_{i p}^{\alpha} R_{p j k m, m}+2 h_{i j m}^{\delta} R_{\delta \alpha k m}+h_{i j}^{\delta} R_{\delta \alpha k m, m}
\end{aligned}
$$

In the following we will choose an orthonormal frame field on the closed surfaces minimally immersed in a unit sphere, under which the shape operators have very simple forms.

If the normal bundle of $M$ immersed in $S^{n}$ is flat, the shape operator $L^{\alpha}$ with respect to $e_{\alpha}$ can be diagonalized simultaneously for $\alpha=3, \cdots, n$. Otherwise, at least one of $h_{12}^{\beta}$ is not zero. Choosing a unit normal vector field $\widetilde{e}_{3}=e /|e|$ where $e=\sum_{\beta=3}^{n} h_{12}^{\beta} e_{\beta}$, and taking an orthogonal transformation in the normal space $N_{x}(M)$, we have

$$
\left(\begin{array}{c}
\widetilde{e}_{3} \\
\widetilde{e}_{4} \\
\vdots \\
\widetilde{e}_{n}
\end{array}\right)=\left(\begin{array}{cccc}
h_{12}^{3}|e|^{-1} & h_{12}^{4}|e|^{-1} & \ldots & h_{12}^{n}|e|^{-1} \\
a_{43} & a_{44} & \ldots & a_{4 n} \\
\vdots & \vdots & & \vdots \\
a_{n 3} & a_{n 4} & \ldots & a_{n n}
\end{array}\right)\left(\begin{array}{c}
e_{3} \\
e_{4} \\
\vdots \\
e_{n}
\end{array}\right) .
$$

Let $\widetilde{L}^{\alpha}=\left(\widetilde{h}_{i j}^{\alpha}\right)$ be the shape operator with respect to $\widetilde{e}_{\alpha}$. Then 


$$
\left\{\begin{array}{l}
h_{i j}^{3}=h_{12}^{3}|e|^{-1} \widetilde{h}_{i j}^{3}+a_{43} \widetilde{h}_{i j}^{4}+\cdots+a_{n 3} \widetilde{h}_{i j}^{n}, \\
h_{i j}^{4}=h_{12}^{4}|e|^{-1} \widetilde{h}_{i j}^{3}+a_{44} \widetilde{h}_{i j}^{4}+\cdots+a_{n 4} \widetilde{h}_{i j}^{n}, \\
\cdots \ldots \ldots \ldots \ldots \ldots \ldots \ldots \ldots \ldots \ldots \ldots \ldots \ldots \ldots \ldots \\
h_{i j}^{n}=h_{12}^{n}|e|^{-1} \widetilde{h}_{i j}^{3}+a_{4 n} \widetilde{h}_{i j}^{4}+\cdots+a_{n n} \widetilde{h}_{i j}^{n} .
\end{array}\right.
$$

Put $i=1$ and $j=2$ in $(*)$. Then $(*)$ has a unique solution

$$
b:=\widetilde{h}_{12}^{3}=|e| \neq 0, \quad \widetilde{h}_{12}^{\beta}=0, \quad 4 \leq \beta \leq n .
$$

We denote $\lambda^{\beta}=\tilde{h}_{11}^{\beta}$ for $\beta \geq 3$. So the shape operators $\tilde{L}^{\alpha}$ have the following forms:

$$
\tilde{L}^{3}=\left(\begin{array}{cc}
\lambda^{3} & b \\
b & \nu^{3}
\end{array}\right), \quad \tilde{L}^{\beta}=\left(\begin{array}{cc}
\lambda^{\beta} & 0 \\
0 & \nu^{\beta}
\end{array}\right),
$$

where $\beta=4, \cdots, n$.

When $M$ is a minimal surface immersed in $S^{n}$, then

$$
\lambda^{\alpha}+\nu^{\alpha}=0,
$$

for $\alpha=3, \cdots, n$. For convenience, we still denote the new frame filed by $\left\{e_{\alpha}\right\}$ and the corresponding second fundamental form by $\left\{h_{i j}^{\alpha}\right\}$. We denote

$$
\bar{S}:=\sum_{(i, j, \beta>3)}\left(h_{i j}^{\beta}\right)^{2}=2 \sum_{(\beta>3)}\left(\lambda^{\beta}\right)^{2}, \quad S_{3}:=\sum_{(i, j)}\left(h_{i j}^{3}\right)^{2}=2\left(\lambda^{3}\right)^{2}+2 b^{2} .
$$

Then

$$
S=\bar{S}+S_{3}=2 \sum_{(\beta>3)}\left(\lambda^{\beta}\right)^{2}+2\left(\lambda^{3}\right)^{2}+2 b^{2} .
$$

According to (2.7), we define

$$
\lambda_{i}^{\alpha}:=h_{11 i}^{\alpha}=-h_{22 i}^{\alpha}, \quad \lambda_{i j}^{\alpha}:=h_{11 i j}^{\alpha}=-h_{22 i j}^{\alpha} .
$$

By the symmetry of $h_{i j k}^{\alpha}$ and $h_{i j k l}^{\alpha}$ with respect to indices $i, j, k$, we denote

$$
\begin{aligned}
P & :=\sum\left(h_{i j k}^{\alpha}\right)^{2}=4 \sum_{\alpha=3}^{n}\left(\left(\lambda_{1}^{\alpha}\right)^{2}+\left(\lambda_{2}^{\alpha}\right)^{2}\right), \\
Q & :=\sum\left(h_{i j k l}^{\alpha}\right)^{2}=4 \sum_{\alpha=3}^{n}\left(\left(\lambda_{11}^{\alpha}\right)^{2}+\left(\lambda_{22}^{\alpha}\right)^{2}+\left(\lambda_{12}^{\alpha}\right)^{2}+\left(\lambda_{21}^{\alpha}\right)^{2}\right) .
\end{aligned}
$$

It follows from (2.1) and (2.6) that the Riemannian curvature tensor, the normal curvature tensor and the first covariant differentials of the normal curvature tensor become

$$
\begin{gathered}
R_{i j k l}=(1-S / 2)\left(\delta_{i k} \delta_{j l}-\delta_{i l} \delta_{j k}\right), \quad R_{3 \beta 12}=-2 b \lambda^{\beta}, \quad R_{\gamma \beta 12}=0, \\
R_{3 \beta 12, k}=2\left(\lambda^{3} h_{12 k}^{\beta}-\lambda^{\beta} h_{12 k}^{3}-b \lambda_{k}^{\beta}\right), \quad R_{\beta \gamma 12, k}=2\left(\lambda^{\beta} h_{12 k}^{\gamma}-\lambda^{\gamma} h_{12 k}^{\beta}\right),
\end{gathered}
$$

where $\beta, \gamma=4, \cdots, n$. It is not difficult to check that

$$
S_{k}=2 \sum h_{i j}^{\alpha} h_{i j k}^{\alpha}=4 \sum_{\beta=4}^{n} \lambda^{\beta} \lambda_{k}^{\beta}+4\left(\lambda^{3} \lambda_{k}^{3}+b h_{12 k}^{3}\right) .
$$

Hence

$$
\frac{1}{4} S_{1}=\sum_{\beta=4}^{n} \lambda^{\beta} \lambda_{1}^{\beta}+\lambda^{3} \lambda_{1}^{3}+b \lambda_{2}^{3}, \quad \frac{1}{4} S_{2}=\sum_{\beta=4}^{n} \lambda^{\beta} \lambda_{2}^{\beta}+\lambda^{3} \lambda_{2}^{3}-b \lambda_{1}^{3} .
$$


From Ricci's formula (2.3), we have

$$
\begin{array}{ll}
\lambda_{12}^{3}-\lambda_{21}^{3}=-(2-S-\bar{S}) b, & \lambda_{11}^{3}+\lambda_{22}^{3}=(2-S) \lambda^{3}, \\
\lambda_{11}^{\beta}+\lambda_{22}^{\beta}=\left(2-S-2 b^{2}\right) \lambda^{\beta}, & \lambda_{12}^{\beta}-\lambda_{21}^{\beta}=-2 b \lambda^{3} \lambda^{\beta},
\end{array}
$$

for $\beta=4, \cdots, n$.

Using the above formulae, we can obtain the following proposition for later use.

Proposition 2.1. Let $M$ be a surface minimally immersed in a unit sphere $S^{n}$. Then

$$
\frac{1}{2} \Delta S=P+(2-S) S-4 b^{2} \bar{S} .
$$

Proof. From (2.4) and (2.10), we have

$$
\begin{aligned}
\sum_{i, j, \alpha} h_{i j}^{\alpha} \Delta h_{i j}^{\alpha} & =\sum_{i, j, p, m, \alpha}\left(h_{i j}^{\alpha} h_{p i}^{\alpha} R_{p m j m}+h_{i j}^{\alpha} h_{m p}^{\alpha} R_{p i j m}\right)+\sum_{i, j, m, \alpha, \delta} h_{i j}^{\alpha} h_{m i}^{\delta} R_{\delta \alpha j m} \\
& =(2-S)\left(h_{i j}^{\alpha}\right)^{2}+\sum_{\beta=4}^{n} 4 b \lambda^{\beta} R_{3 \beta 12}=(2-S) S-4 b^{2} \bar{S}
\end{aligned}
$$

Hence

$$
\frac{1}{2} \Delta S=\sum_{\alpha, i, j, k}\left(h_{i j k}^{\alpha}\right)^{2}+\sum_{\alpha, i, j} h_{i j}^{\alpha} \Delta h_{i j}^{\alpha}=P+(2-S) S-4 b^{2} \bar{S} .
$$

Remark 2.2. If the normal bundle of the surface $M$ minimally immersed in $S^{n}$ is flat, we choose $e_{1}, e_{2}$ such that $b$ is zero. We easily get

$$
\frac{1}{2} \Delta S=P+(2-S) S .
$$

Next we consider the case that the normal bundle of $M$ is nowhere flat. In this case, $b \neq 0$ and we can establish the following Theorem 2.3 Some partial result was obtained in [8]. Here, we will give the detailed proof of the theorem for the completeness.

Theorem 2.3. Let $M$ be a surface minimally immersed in a unit sphere $S^{n}$ with nowhere flat normal bundle. If the Gauss curvature of $M$ is positive, we can establish a local orthonormal frame filed $\left\{e_{3}, \cdots, e_{n}\right\}$ normal to $M$ such that the shape operators $L^{\alpha}$ with respect to $e_{\alpha}$ have the following forms:

$$
L^{3}=\left(\begin{array}{cc}
0 & b \\
b & 0
\end{array}\right), \quad L^{4}=\left(\begin{array}{cc}
b & 0 \\
0 & -b
\end{array}\right), \quad L^{\beta}=\left(\begin{array}{ll}
0 & 0 \\
0 & 0
\end{array}\right),
$$

where $\beta=5, \cdots, n$. Furthermore

$$
\begin{aligned}
& b^{2}=S / 4, \quad \lambda_{1}^{3}=-\lambda_{2}^{4}=-\frac{1}{4 \sqrt{S}} S_{2}, \quad \lambda_{2}^{3}=\lambda_{1}^{4}=\frac{1}{4 \sqrt{S}} S_{1}, \\
& \lambda_{11}^{3}=-\lambda_{21}^{4}=-\frac{1}{4 \sqrt{S}} S_{21}, \quad \lambda_{12}^{3}=-\lambda_{22}^{4}=-\frac{1}{4 \sqrt{S}}\left(S_{22}-P\right), \\
& \lambda_{22}^{3}=\lambda_{12}^{4}=\frac{1}{4 \sqrt{S}} S_{12}, \quad \lambda_{21}^{3}=\lambda_{11}^{4}=\frac{1}{4 \sqrt{S}}\left(S_{11}-P\right) .
\end{aligned}
$$


Proof. We take the orthonormal frame field $\left\{e_{1}, e_{2}, e_{3}, \cdots, e_{n}\right\}$ on $M$ such that the shape operators have the form

$$
L^{3}=\left(\begin{array}{cc}
\lambda^{3} & b \\
b & -\lambda^{3}
\end{array}\right) ; \quad L^{\beta}=\left(\begin{array}{cc}
\lambda^{\beta} & 0 \\
0 & -\lambda^{\beta}
\end{array}\right)
$$

where $\beta=4, \cdots, n$. It is easy to check from (2.4) that

$$
\begin{aligned}
\sum_{i, j, k, \alpha}\left(h_{i j k}^{\alpha} \Delta h_{i j}^{\alpha}\right)_{k} & =\sum_{i, j, \alpha}\left(\Delta h_{i j}^{\alpha}\right)^{2}+\sum_{i, j, k, l, p, \alpha}\left(h_{i j k}^{\alpha} h_{p i k}^{\alpha} R_{p l j l}+h_{i j k}^{\alpha} h_{l p k}^{\alpha} R_{p i j l}\right) \\
& +\sum_{i, j, k, l, p, \alpha}\left(h_{i j k}^{\alpha} h_{p i}^{\alpha} R_{p l j l, k}+h_{i j k}^{\alpha} h_{l p}^{\alpha} R_{p i j l, k}\right) \\
& +\sum_{i, j, k, l, \alpha, \delta} h_{i j k}^{\alpha} h_{l i k}^{\delta} R_{\delta \alpha j l}+\sum_{i, j, k, l, \alpha, \delta} h_{i j k}^{\alpha} h_{l i}^{\delta} R_{\delta \alpha j l, k} .
\end{aligned}
$$

Firstly, by (2.4) and (2.10), we get

$$
\begin{aligned}
& \Delta h_{11}^{\beta}=\left(2-S-2 b^{2}\right) \lambda^{\beta}, \quad \Delta h_{12}^{\beta}=2 b \lambda^{3} \lambda^{\beta}, \\
& \Delta h_{12}^{3}=(2-S-\bar{S}) b, \quad \Delta h_{11}^{3}=(2-S) \lambda^{3},
\end{aligned}
$$

for $\beta=4, \cdots n$, so

$$
\begin{aligned}
\sum_{i, j, \alpha}\left(\Delta h_{i j}^{\alpha}\right)^{2} & =2 \sum_{\beta=4}^{n}\left(\Delta h_{11}^{\beta}\right)^{2}+2\left(\Delta h_{11}^{3}\right)^{2}+2 \sum_{\beta=4}^{n}\left(\Delta h_{12}^{\beta}\right)^{2}+\left(\Delta h_{12}^{3}\right)^{2} \\
& =(2-S)^{2} S+2(5 S-8) b^{2} \bar{S} .
\end{aligned}
$$

Secondly, using (2.10) and (2.12), we get

$$
\begin{aligned}
\sum_{i, j, k, l, p, \alpha}\left(h_{i j k}^{\alpha} h_{p i k}^{\alpha} R_{p l j l}\right. & \left.+h_{i j k}^{\alpha} h_{l p k}^{\alpha} R_{p i j l}\right)+\sum_{i, j, k, l, \alpha, \delta} h_{i j k}^{\alpha} h_{l i k}^{\delta} R_{\delta \alpha j l} \\
& =(2-S) P+\sum_{\gamma=4}^{n} 8\left(\lambda_{1}^{3} \lambda_{2}^{\gamma}-\lambda_{2}^{3} \lambda_{1}^{\gamma}\right) R_{\gamma 312} \\
& =(2-S) P+16 b \sum_{\gamma=4}^{n}\left(\lambda_{1}^{3} \lambda^{\gamma} \lambda_{2}^{\gamma}-\lambda_{2}^{3} \lambda^{\gamma} \lambda_{1}^{\gamma}\right) . \\
& =(2-S) P+4 b^{2} \sum_{i, j, k}\left(h_{i j k}^{3}\right)^{2}+4 b\left(\lambda_{1}^{3} S_{2}-\lambda_{2}^{3} S_{1}\right) .
\end{aligned}
$$

Thirdly, by the first formula of (2.10), we have

$$
\sum_{i, j, k, l, p, \alpha}\left(h_{i j k}^{\alpha} h_{p i}^{\alpha} R_{p l j l, k}+h_{i j k}^{\alpha} h_{l p}^{\alpha} R_{p i j l, k}\right)=-\sum_{i, j, k, \alpha} h_{i j}^{\alpha} h_{i j k}^{\alpha} S_{k}=-\frac{1}{2}|\nabla S|^{2} .
$$

At last, using (2.11), we have

$$
\begin{aligned}
\sum_{i, j, k, l, \alpha, \delta} h_{i j k}^{\alpha} h_{l i}^{\delta} R_{\delta \alpha j l, k} & =\sum_{\gamma=4}^{n} 2\left(\lambda^{\gamma} \lambda_{2}^{3}+b \lambda_{1}^{\gamma}-\lambda^{3} \lambda_{2}^{\gamma}\right) R_{3 \gamma 12,1} \\
& +\sum_{\gamma=4}^{n} 2\left(-\lambda^{\gamma} \lambda_{1}^{3}+b \lambda_{2}^{\gamma}+\lambda^{3} \lambda_{1}^{\gamma}\right) R_{3 \gamma 12,2} \\
& +\sum_{\beta, \gamma=4}^{n}\left(-2 \lambda^{\gamma} \lambda_{2}^{\beta} R_{\gamma \beta 12,1}+2 \lambda^{\gamma} \lambda_{1}^{\beta} R_{\gamma \beta 12,2}\right) . \\
& =-\frac{1}{2} S P+4 b^{2} \sum_{i, j, k}\left(h_{i j k}^{3}\right)^{2}+4 b\left(\lambda_{1}^{3} S_{2}-\lambda_{2}^{3} S_{1}\right)+\frac{1}{4}|\nabla S|^{2} .
\end{aligned}
$$


Substituting (2.19), (2.20),(2.21) and (2.22) into (2.18), we have

$$
\begin{aligned}
\sum_{i, j, k, \alpha}\left(h_{i j k}^{\alpha} \Delta h_{i j}^{\alpha}\right)_{k} & =\left(2-\frac{3}{2} S\right) P+(2-S)^{2} S+2(5 S-8) b^{2} \bar{S} \\
& +8 b^{2} \sum_{i, j, k}\left(h_{i j k}^{3}\right)^{2}+8 b\left(\lambda_{1}^{3} S_{2}-\lambda_{2}^{3} S_{1}\right)-\frac{1}{4}|\nabla S|^{2},
\end{aligned}
$$

which together with $P=\frac{1}{2} \Delta S-(2-S) S+4 b^{2} \bar{S}$ and $S \Delta S=\frac{1}{2} \Delta S^{2}-|\nabla S|^{2}$ forces that

$$
\begin{aligned}
\sum_{i, j, k, \alpha}\left(h_{i j k}^{\alpha} \Delta h_{i j}^{\alpha}\right)_{k}= & \frac{1}{2}(2-S) S^{2}+4(S-2) b^{2} \bar{S}+\Delta S-\frac{3}{8} \Delta S^{2} \\
& +8 b^{2} \sum_{i, j, k}\left(h_{i j k}^{3}\right)^{2}+8 b\left(\lambda_{1}^{3} S_{2}-\lambda_{2}^{3} S_{1}\right)+\frac{1}{2}|\nabla S|^{2} .
\end{aligned}
$$

Taking integration over $M$ on both sides of (2.24), we have

$$
\int_{M}\left\{\frac{1}{2} S^{2}(2-S)+4(S-2) b^{2} \bar{S}+2\left(4 b \lambda_{1}^{3}+\frac{1}{2} S_{2}\right)^{2}+2\left(4 b \lambda_{2}^{3}-\frac{1}{2} S_{1}\right)^{2}\right\}=0,
$$

that is

$$
\begin{aligned}
\int_{M}(2-S) b^{2} \bar{S} & =\int_{M}\left\{\frac{1}{8} S^{2}(2-S)+\frac{1}{2}\left(4 b \lambda_{1}^{3}+\frac{1}{2} S_{2}\right)^{2}+\frac{1}{2}\left(4 b \lambda_{2}^{3}-\frac{1}{2} S_{1}\right)^{2}\right\} \\
& \geq \int_{M} \frac{1}{8} S^{2}(2-S)
\end{aligned}
$$

and the equality holds if and only if

$$
b \lambda_{1}^{3}=-\frac{1}{8} S_{2}, \quad b \lambda_{2}^{3}=\frac{1}{8} S_{1} .
$$

On the other hand, it is easy to check

$$
b^{2} \bar{S} \leq \frac{1}{2} S_{3} \bar{S} \leq \frac{1}{8}\left(S_{3}+\bar{S}\right)^{2}=\frac{1}{8} S^{2},
$$

and the equality holds if and only if

$$
\lambda^{3}=0, \quad \bar{S}=S_{3}=2 b^{2} .
$$

Since the Gauss curvature of $M$ is positive, we have $S<2$. Taking integration over $M$ on both sides of (2.26), we obtain

$$
\int_{M}(2-S) b^{2} \bar{S} \leq \int_{M} \frac{1}{8} S^{2}(2-S) .
$$

It follows from (2.25) and (2.27) that

$$
\int_{M}(2-S) b^{2} \bar{S}=\int_{M} \frac{1}{8} S^{2}(2-S)
$$

which implies that the equalities in (2.25) and (2.26) hold always. Therefore

$$
\lambda^{3}=0, \quad b \lambda_{1}^{3}=-\frac{1}{8} S_{2}, \quad b \lambda_{2}^{3}=\frac{1}{8} S_{1}, \quad \bar{S}=2 b^{2} .
$$

This together with the fact that $S=\bar{S}+2 b^{2}$ yields

$$
b^{2}=\frac{1}{4} S, \quad \bar{S}=\frac{1}{2} S, \quad \lambda_{1}^{3}=\frac{1}{4 \sqrt{S}} S_{2}, \quad \lambda_{2}^{3}=\frac{1}{4 \sqrt{S}} S_{1} .
$$

Therefore we deduce that there must exist a number $\beta$ such that $\lambda^{\beta} \neq 0$ for $\beta \geq 4$. We choose a unit normal vector field $\bar{e}_{4}=e /|e|$ where $e=\sum_{\gamma=4}^{n} h_{11}^{\gamma} e_{\gamma}$, and take an orthogonal transformation in the normal space $N_{x}(M): \bar{e}_{3}=e_{3}$ and 


$$
\left(\begin{array}{c}
\bar{e}_{4} \\
\bar{e}_{5} \\
\vdots \\
\bar{e}_{n}
\end{array}\right)=\left(\begin{array}{cccc}
h_{11}^{4}|e|^{-1} & h_{11}^{4}|e|^{-1} & \cdots & h_{11}^{n}|e|^{-1} \\
b_{54} & b_{55} & \ldots & b_{5 n} \\
\vdots & \vdots & & \vdots \\
b_{n 4} & b_{n 5} & \ldots & b_{n n}
\end{array}\right)\left(\begin{array}{c}
e_{4} \\
e_{5} \\
\vdots \\
e_{n}
\end{array}\right) .
$$

Let $\bar{L}^{\alpha}=\left(\bar{h}_{i j}^{\alpha}\right)$ be the shape operators with respect to $\bar{e}_{\alpha}, 3 \leq \alpha \leq n$. It follows that

$$
\left\{\begin{array}{l}
h_{i j}^{3}=\bar{h}_{i j}^{3}, \\
h_{i j}^{4}=\bar{h}_{11}^{4}|e|^{-1} \bar{h}_{i j}^{4}+b_{54} \bar{h}_{i j}^{5}+\cdots+b_{n 4} \bar{h}_{i j}^{n}, \\
h_{i j}^{5}=\bar{h}_{11}^{5}|e|^{-1} \bar{h}_{i j}^{4}+b_{55} \bar{h}_{i j}^{5}+\cdots+b_{n 5} \bar{h}_{i j}^{n}, \\
\ldots \ldots \ldots \ldots \ldots \ldots \ldots \ldots \ldots \ldots \ldots \ldots \ldots \\
h_{i j}^{n}=\bar{h}_{11}^{n}|e|^{-1} \bar{h}_{i j}^{4}+b_{5 n} \bar{h}_{i j}^{5}+\cdots+b_{n n} \bar{h}_{i j}^{n} .
\end{array}\right.
$$

Put $i=1$ and $j=1$ in $(\star)$. Then it is easy to check that $(\star)$ has unique solution

$$
\bar{h}_{11}^{4}=|e|^{-1}>0, \quad \bar{h}_{11}^{\gamma}=0, \quad 5 \leq \gamma \leq n .
$$

Put $i=1$ and $j=2$ in $(\star)$. Then it is easy to check that $(\star)$ has unique solution $\bar{h}_{12}^{\gamma}=0,5 \leq \gamma \leq n$. Therefore the shape operators with respect to $\left\{e_{1}, e_{2} ; \bar{e}_{\gamma}\right\}_{\gamma=3}^{n}$ have the following forms:

$$
\bar{L}^{3}=\left(\begin{array}{cc}
0 & b \\
b & 0
\end{array}\right), \quad \bar{L}^{4}=\left(\begin{array}{cc}
\bar{\lambda}^{4} & 0 \\
0 & -\bar{\lambda}^{4}
\end{array}\right), \quad \bar{L}^{\beta}=\left(\begin{array}{cc}
0 & 0 \\
0 & 0
\end{array}\right), \quad 5 \leq \beta \leq n,
$$

where $b^{2}=\left(\bar{\lambda}^{4}\right)^{2}=S / 4$, that is $\bar{\lambda}^{4}=b=\sqrt{S} / 2$. For convenience, we denote the new frame field by $\left\{e_{1}, e_{2} ; e_{\gamma}\right\}_{\gamma=3}^{n}$. So far, we have built a frame field on $M$ such that the shape operators have the following forms:

$$
L^{3}=\left(\begin{array}{cc}
0 & b \\
b & 0
\end{array}\right), \quad L^{4}=\left(\begin{array}{cc}
b & 0 \\
0 & -b
\end{array}\right), \quad L^{\beta}=\left(\begin{array}{cc}
0 & 0 \\
0 & 0
\end{array}\right), \quad 5 \leq \beta \leq n .
$$

Furthermore

$$
b^{2}=S / 4, \quad \lambda_{1}^{3}=-\frac{1}{4 \sqrt{S}} S_{2}, \quad \lambda_{2}^{3}=\frac{1}{4 \sqrt{S}} S_{1} .
$$

It follows from Chern [4] and the choice of the normal vector field $e_{3}, e_{4}$ that

$$
\sum_{\gamma=5}^{n} \lambda_{1}^{\gamma} \lambda_{2}^{\gamma}=0, \quad \sum_{\gamma=5}^{n}\left(\left(\lambda_{1}^{\gamma}\right)^{2}-\left(\lambda_{2}^{\gamma}\right)^{2}\right)=0
$$

Next we take covariant differential of $h_{11}^{4}$ and have

$$
h_{11 k}^{4} \omega_{k}=d h_{11}^{4}+2 h_{12}^{4} \omega_{21}+\sum_{\alpha=3}^{n} h_{11}^{\alpha} \omega_{\alpha 4}=d h_{11}^{4}=\frac{1}{4 \sqrt{S}} S_{k} \omega_{k},
$$

which implies

$$
\lambda_{1}^{4}=\lambda_{2}^{3}=\frac{1}{4 \sqrt{S}} S_{1}, \quad \lambda_{2}^{4}=-\lambda_{1}^{3}=\frac{1}{4 \sqrt{S}} S_{2} .
$$


We take covariant differential of $h_{11}^{\gamma}$ and $h_{12}^{\gamma}$ for $5 \leq \gamma \leq n$,

$$
\begin{aligned}
& h_{11 k}^{\gamma} \omega_{k}=d h_{11}^{\gamma}+2 h_{12}^{\gamma} \omega_{21}+\sum_{\alpha=3}^{n} h_{11}^{\alpha} \omega_{\alpha \gamma}=b \omega_{4 \gamma}, \\
& h_{12 k}^{\gamma} \omega_{k}=d h_{12}^{\gamma}+h_{22}^{\gamma} \omega_{21}+h_{11}^{\gamma} \omega_{12}+\sum_{\alpha=3}^{n} h_{12}^{\alpha} \omega_{\alpha \gamma}=b \omega_{3 \gamma},
\end{aligned}
$$

which imply

$$
\omega_{3 \gamma}=\frac{1}{b}\left(\lambda_{2}^{\gamma} \omega_{1}-\lambda_{1}^{\gamma} \omega_{2}\right), \quad \omega_{4 \gamma}=\frac{1}{b}\left(\lambda_{1}^{\gamma} \omega_{1}+\lambda_{2}^{\gamma} \omega_{2}\right) .
$$

We take covariant differential of $h_{111}^{3}, h_{112}^{3}, h_{111}^{4}$ and $h_{112}^{4}$ respectively

$$
\begin{aligned}
& h_{111 k}^{3} \omega_{k}=d h_{111}^{3}+3 h_{211}^{3} \omega_{21}+h_{111}^{4} \omega_{43}+\sum_{\gamma=5}^{n} h_{111}^{\gamma} \omega_{\gamma 3}, \\
& h_{112 k}^{3} \omega_{k}=d h_{112}^{3}+2 h_{212}^{3} \omega_{21}+h_{111}^{3} \omega_{12}+h_{112}^{4} \omega_{43}+\sum_{\gamma=5}^{n} h_{112}^{\gamma} \omega_{\gamma 3}, \\
& h_{111 k}^{4} \omega_{k}=d h_{111}^{4}+3 h_{211}^{4} \omega_{21}+h_{111}^{3} \omega_{34}+\sum_{\gamma=5}^{n} h_{111}^{\gamma} \omega_{\gamma 4}, \\
& h_{112 k}^{4} \omega_{k}=d h_{112}^{4}+2 h_{212}^{4} \omega_{21}+h_{111}^{4} \omega_{12}+h_{112}^{3} \omega_{34}+\sum_{\gamma=5}^{n} h_{112}^{\gamma} \omega_{\gamma 4} .
\end{aligned}
$$

Then from (2.32), (2.35) and use (2.30), (2.29) we have

$$
\begin{aligned}
\left(\lambda_{11}^{3}+\lambda_{21}^{4}\right) \omega_{1} & +\left(\lambda_{12}^{3}+\lambda_{22}^{4}\right) \omega_{2}=\sum_{\gamma=5}^{n}\left(\lambda_{1}^{\gamma} \omega_{\gamma 3}+\lambda_{2}^{\gamma} \omega_{\gamma 4}\right) \\
& =-\frac{2}{b} \sum_{\gamma=5}^{n} \lambda_{1}^{\gamma} \lambda_{2}^{\gamma} \omega_{1}+\frac{1}{b} \sum_{\gamma=5}^{n}\left\{\left(\lambda_{1}^{\gamma}\right)^{2}-\left(\lambda_{2}^{\gamma}\right)^{2}\right\} \omega_{2}=0 .
\end{aligned}
$$

It follows from (2.33), (2.34), (2.30) and (2.29) that

$$
\begin{aligned}
\left(\lambda_{21}^{3}-\lambda_{11}^{4}\right) \omega_{1} & +\left(\lambda_{22}^{3}-\lambda_{12}^{4}\right) \omega_{2}=\sum_{\gamma=5}^{n}\left(\lambda_{2}^{\gamma} \omega_{\gamma 3}-\lambda_{1}^{\gamma} \omega_{\gamma 4}\right) \\
& =\frac{2}{b} \sum_{\gamma=5}^{n} \lambda_{1}^{\gamma} \lambda_{2}^{\gamma} \omega_{2}+\frac{1}{b} \sum_{\gamma=5}^{n}\left(\left(\lambda_{1}^{\gamma}\right)^{2}-\left(\lambda_{2}^{\gamma}\right)^{2}\right) \omega_{1}=0
\end{aligned}
$$

therefore

$$
\lambda_{11}^{3}+\lambda_{21}^{4}=0, \quad \lambda_{12}^{3}+\lambda_{22}^{4}=0, \quad \lambda_{21}^{3}-\lambda_{11}^{4}=0, \quad \lambda_{22}^{3}-\lambda_{12}^{4}=0 .
$$

On the other hand, we study the second covariant differentials of $S$. It is not difficult to check that for all $k=1,2$,

$$
S_{k l}=2 \sum\left(h_{i j l}^{\alpha} h_{i j k}^{\alpha}+h_{i j}^{\alpha} h_{i j k l}^{\alpha}\right)=4 \sum_{\alpha}\left(\lambda_{k}^{\alpha} \lambda_{l}^{\alpha}+h_{12 k}^{\alpha} h_{12 l}^{\alpha}\right)+b \lambda_{k l}^{3}+b h_{12 k l}^{4},
$$

which is equal to

$$
\begin{array}{ll}
\lambda_{11}^{4}+\lambda_{21}^{3}=\frac{1}{2 \sqrt{S}}\left(S_{11}-P\right), & \lambda_{12}^{4}+\lambda_{22}^{3}=\frac{1}{2 \sqrt{S}} S_{12} \\
\lambda_{22}^{4}-\lambda_{12}^{3}=\frac{1}{2 \sqrt{S}}\left(S_{22}-P\right), & \lambda_{21}^{4}-\lambda_{11}^{3}=\frac{1}{2 \sqrt{S}} S_{21} .
\end{array}
$$

This together with (2.36) gives (2.16). So we complete the proof of Theorem 2.3. 
From now on we use the orthonormal frame field established by Theorem 2.3 . We conclude this section with some interesting and elementary formulas which will be useful in the next section. Firstly, it follows from Theorem 2.3 that $b^{2} \bar{S}=S^{2} / 8$. So we can rewrite Proposition 2.1 as follows.

Proposition 2.4. Suppose that $M$ is a closed surface minimally immersed in a unit sphere $S^{n}$ with positive Gauss curvature and nowhere flat normal bundle. We have

$$
\frac{1}{2} \Delta S=P-\frac{1}{2} S(3 S-4)
$$

The Riemannian curvature tensor, the normal curvature tensor and the first covariant differentials of the normal curvature tensor in (2.10) and (2.11) can be simplified as

$$
\begin{gathered}
R_{i j k l}=\left(1-\frac{S}{2}\right)\left(\delta_{i k} \delta_{j l}-\delta_{i l} \delta_{j k}\right), \quad R_{i j k l, m}=-\frac{1}{2} S_{m}\left(\delta_{i k} \delta_{j l}-\delta_{i l} \delta_{j k}\right) \\
R_{3412}=-\frac{1}{2} S, \quad R_{3 \beta 12}=R_{4 \beta 12}=R_{\beta \gamma 12}=0 \\
R_{3412, k}=-\frac{1}{2} S_{k}, \quad R_{3 \beta 12,1}=-2 b \lambda_{1}^{\beta}, \quad R_{3 \beta 12,2}=-2 b \lambda_{2}^{\beta}, \\
R_{\beta \gamma 12, k}=0, \quad R_{4 \beta 12,2}=-2 b \lambda_{1}^{\beta}, \quad R_{4 \beta 12,1}=2 b \lambda_{2}^{\beta}
\end{gathered}
$$

for $5 \leq \beta, \gamma \leq n$. The Ricci's formula in (2.13) becomes

$$
\begin{array}{ll}
\lambda_{11}^{3}+\lambda_{22}^{3}=0, & \lambda_{12}^{3}-\lambda_{21}^{3}=\frac{1}{4} \sqrt{S}(3 S-4), \\
\lambda_{11}^{\beta}+\lambda_{22}^{\beta}=0, & \lambda_{12}^{\beta}-\lambda_{21}^{\beta}=0
\end{array}
$$

for $5 \leq \beta \leq n$.

\section{Main Results}

In 3], Calabi considered minimal immersions of compact surfaces without boundary and with constant Gauss curvature $K$ into $S^{n}$. He gave a complete list of all such immersions and proved that the set of possible values of $K$ is discrete, namely $K=K(s)=2 /(s(s+1)), s \in \mathbb{N}$. This led to the Simon conjecture as follows (see [15]).

Simon conjecture (intrinsic version): Let $M$ be a compact surface minimally immersed into $S^{n}$. If $K(s+1) \leq K \leq K(s)$ for an $s \in \mathbb{N}$, then either $K=K(s+1)$ or $K=K(s)$ and the immersion is one of the Calabi's standard minimal immersion.

There is another version of this conjecture for the extrinsic curvature functions $S$. For minimal surfaces in $S^{n}$, both curvature functions are related as follows:

$$
2 K=2-S, \quad S=\frac{2(s-1)(s+2)}{s(s+1)}, \quad s \in \mathbb{N} .
$$

Thus, for Calabi's standard immersions, we have 
Simon conjecture (extrinsic version): Let $M$ be a compact surface minimally immersed into $S^{n}$. If

$$
\frac{2(s-1)(s+2)}{s(s+1)} \leq S \leq \frac{2 s(s+3)}{(s+1)(s+2)}, \quad s \in \mathbb{N}
$$

then either $S=\frac{2(s-1)(s+2)}{s(s+1)}$ or $S=\frac{2 s(s+3)}{(s+1)(s+2)}$, and the immersion is one of the Calabi's standard minimal immersion.

For a minimal immersion as considered above, $K=K(s)=1$ for $s=1$ gives $S=0$, and the immersion is an equator in $S^{3}(1) . K=K(s)=\frac{1}{3}$ for $s=2$ gives $S=\frac{4}{3}$ and the immersion is a Veronese surface in $S^{4}(1) . K=K(s)=\frac{1}{6}$ for $s=3$ gives $S=\frac{5}{3}$ and the immersion is a generalized Veronese surface in $S^{6}(1)$.

So far, Simon conjecture has been solved in the case $s=1$ and $s=2$, see [2,11,12]. Using the frame field established by Theorem 2.3, we give a very simple proof of Simon conjecture for the minimal surface in $S^{n}$ with flat or nowhere flat normal bundle, which is critical for later use.

Theorem 3.1. Let $M$ be a closed minimal surface immersed in $S^{n}$ with flat or nowhere flat normal bundle. If $0 \leq S \leq 4 / 3$, then $S=0$ or $S=4 / 3$.

Proof. If the normal bundle is flat, it follows from Remark 2.2 and $0 \leq S \leq 4 / 3$ that

$$
\frac{1}{2} \Delta S=P+(2-S) S \geq(2-S) S \geq 0
$$

By integration, we have $S=0$.

If the normal bundle is nowhere flat, the assumption $0 \leq S \leq 4 / 3$ implies that the Gauss curvature of the minimal surface is positive. It follows from (2.37) in Proposition 2.4 that

$$
\frac{1}{2} \Delta S=P-\frac{1}{2} S(3 S-4) \geq-\frac{1}{2} S(3 S-4) \geq 0 .
$$

So we have $S(3 S-4)=0$, it follows that $S=4 / 3$. We complete the proof of Theorem 3.1 .

Theorem 3.2. Let $M$ be a closed minimal surface immersed in $S^{n}$ with flat or nowhere flat normal bundle. If $4 / 3 \leq S \leq 5 / 3$, then $S=4 / 3$ or $S=5 / 3$.

Proof. If the normal bundle is flat, from the condition $4 / 3 \leq S \leq 5 / 3$, we have

$$
\frac{1}{2} \Delta S=P+(2-S) S \geq(2-S) S>0 .
$$

By integration, we get a contradiction.

If the normal bundle is nowhere flat, it follows from $4 / 3 \leq S \leq 5 / 3$ that the Gauss curvature is positive. So we can use the frame field introduced in Theorem 2.3. From (2.5), we have

$$
\begin{aligned}
\sum_{i, j, k, \alpha} h_{i j k}^{\alpha} \Delta h_{i j k}^{\alpha} & =\sum_{i, j, k, \alpha}\left(h_{i j k}^{\alpha} \Delta h_{i j}^{\alpha}\right)_{k}-\sum_{i, j, \alpha}\left(\Delta h_{i j}^{\alpha}\right)^{2}+\sum_{i, j, k, p, m, \alpha} 2 h_{i j k}^{\alpha} h_{p j}^{\alpha} R_{p i k m, m} \\
& +\sum_{i, j, k, p, m, \alpha}\left(h_{i j k}^{\alpha} h_{i j p}^{\alpha} R_{p m k m}+4 h_{i j k}^{\alpha} h_{p j m}^{\alpha} R_{p i k m}\right) \\
& +\sum_{i, j, k, m, \alpha, \delta} 2 h_{i j k}^{\alpha} h_{i j m}^{\delta} R_{\delta \alpha k m}+\sum_{i, j, k, m, \alpha, \delta} h_{i j k}^{\alpha} h_{i j}^{\delta} R_{\delta \alpha k m, m} .
\end{aligned}
$$


From (2.19), we have

$$
\sum_{i, j, \alpha}\left(\Delta h_{i j}^{\alpha}\right)^{2}=(2-S)^{2} S+2(5 S-8) b^{2} \bar{S}=\frac{1}{4} S(3 S-4)^{2} .
$$

From (2.38), we have

$$
2 \sum_{i, j, k, p, m, \alpha} h_{i j k}^{\alpha} h_{p j}^{\alpha} R_{p i k m, m}=-\sum_{i, j, k, \alpha} h_{i j}^{\alpha} h_{i j k}^{\alpha} S_{k}=-\frac{1}{2}|\nabla S|^{2},
$$

and

$$
\sum_{i, j, k, p, m, \alpha}\left(4 h_{i j k}^{\alpha} h_{p j m}^{\alpha} R_{p i k m}+h_{i j k}^{\alpha} h_{i j p}^{\alpha} R_{p m k m}\right)=5\left(1-\frac{S}{2}\right) P .
$$

From (2.39), we have

$$
2 \sum_{i, j, k, m, \alpha, \delta} h_{i j k}^{\alpha} h_{i j m}^{\delta} R_{\delta \alpha k m}=16\left(\lambda_{1}^{3} \lambda_{2}^{4}-\lambda_{2}^{3} \lambda_{1}^{4}\right) R_{4312}=-\frac{1}{2}|\nabla S|^{2} .
$$

From (2.40), we have

$$
\sum_{i, j, k, m, \alpha, \delta} h_{i j k}^{\alpha} h_{i j}^{\delta} R_{\delta \alpha k m, m}=-\frac{1}{2} S \sum_{i, j, k} \sum_{\gamma=5}^{n}\left(h_{i j k}^{\gamma}\right)^{2}-\frac{1}{4}|\nabla S|^{2},
$$

which together with

$$
\sum_{i, j, k}\left(h_{i j k}^{3}\right)^{2}+\sum_{i, j, k}\left(h_{i j k}^{4}\right)^{2}=\frac{1}{2 S}|\nabla S|^{2}
$$

forces that

$$
\sum_{i, j, k, m, \alpha, \delta} h_{i j k}^{\alpha} h_{i j}^{\delta} R_{\delta \alpha k m, m}=-\frac{1}{2} S P .
$$

Substituting (3.3), (3.4), (3.5), (3.6) and (3.7) into (3.2), we get

$$
\begin{aligned}
\sum_{i, j, k, \alpha} h_{i j k}^{\alpha} \Delta h_{i j k}^{\alpha} & =\sum_{i, j, k, \alpha}\left(h_{i j k}^{\alpha} \Delta h_{i j}^{\alpha}\right)_{k}+\frac{5}{2} \Delta S-\frac{3}{4} \Delta S^{2} \\
& +\frac{1}{2}|\nabla S|^{2}-\frac{1}{4} S(3 S-4)(9 S-14) .
\end{aligned}
$$

On the other hand, It follows from (2.9) and (2.36) that

$Q=8\left(\left(\lambda_{11}^{3}\right)^{2}+\left(\lambda_{12}^{3}\right)^{2}+\left(\lambda_{21}^{3}\right)^{2}+\left(\lambda_{22}^{3}\right)^{2}\right)+4 \sum_{\beta=5}^{n}\left(\left(\lambda_{11}^{\beta}\right)^{2}+\left(\lambda_{12}^{\beta}\right)^{2}+\left(\lambda_{21}^{\beta}\right)^{2}+\left(\lambda_{22}^{\beta}\right)^{2}\right)$.

It is easy to check that the relative minimal value $\mathrm{Q}$ with the constraint (2.42) is $\frac{1}{4} S(3 S-4)^{2}$, which together with (3.8) forces that

$$
\begin{aligned}
\frac{1}{2} \Delta P & =\sum_{i, j, k, \alpha} h_{i j k}^{\alpha} \Delta h_{i j k}^{\alpha}+\sum_{i, j, k, l, \alpha}\left(h_{i j k l}^{\alpha}\right)^{2} \\
& \geq \sum_{i, j, k, \alpha}\left(h_{i j k}^{\alpha} \Delta h_{i j}^{\alpha}\right)_{k}+\frac{5}{2} \Delta S-\frac{3}{4} \Delta S^{2}-\frac{1}{2} S(3 S-4)(3 S-5) .
\end{aligned}
$$

Taking integration over $M$ on both sides of (3.9) and using the Stokes formula, we have

$$
0 \geq-\int_{M} \frac{1}{2} S(3 S-4)(3 S-5) .
$$

It follows that $S=4 / 3$ or $S=5 / 3$ if $4 / 3 \leq S \leq 5 / 3$. We complete the proof of Theorem 3.2 . 
Now an important result can be obtained instantly as follows.

Theorem 3.3. Let $M$ be a closed minimal surface immersed in $S^{n}$ with positive Gauss curvature $K$ and flat or nowhere flat normal bundle. Then $K+K^{N}=1$, i.e. $M$ is a minimal Wintgen ideal surface.

Proof. If the normal bundle is flat, since the Gauss curvature of $M$ is positive, we have $S<2$. It follows from Remark 2.2 that

$$
\frac{1}{2} \Delta S=P+(2-S) S \geq(2-S) S \geq 0 .
$$

It follows that $S=0$ and $K=1$, so the Gauss curvature $K$ and the normal curvature $K^{N}$ satisfy $K+K^{N}=1$.

If the normal bundle is nowhere flat, it follows from formula (2.39) that the normal curvature $K^{N}=S / 2=1-K$, so $K+K^{N}=1$. This completes the proof of Theorem 3.3 .

Remark 3.4. In submanifolds theory, the famous DDVV inequality is related to the scalar curvature, normal scalar curvature and mean curvature, which is proved by Ge-Tang [7] and Lu [13, independently. Submanifolds are called Wintgen ideal when the equality in the DDVV inequality holds true exactly. By the equality characterization of the DDVV inequality proved by Ge and Tang [7], the shape operators in Theorem 2.3 could attain the equality of DDVV inequality. In this way, Theorem 3.3 can be also deduced.

Remark 3.5. There are also some important properties concerning the sum of the Gauss curvature and normal curvature for closed surfaces immersed in space forms (see [14]).

As we know very well, there are lots of results concerning the pinching of the second fundamental form in a unit sphere $S^{n}$. But there are little results concerning the pinching of the normal curvature. In the following part, we will provide some new results of closed surfaces immersed in arbitrary dimensional unit sphere $S^{n}$ depending on a pinching of the intrinsic and normal curvature.

Theorem 3.6. Let $M$ be a closed surface minimally immersed in $S^{n}$ with flat or nowhere flat normal bundle satisfying $K^{N} \leq 2 K$.

(1) If $K^{N}=0$, then either

(a) $S=0$ and the surface is the geodesic sphere, or

(b) $S=2$ and the surface is the Clifford torus.

(2) If $K^{N} \neq 0$, then $K^{N}=2 K$ and the surface is the Veronese surface in $S^{4}$.

Proof. If the normal bundle is flat, we have $K^{N}=0$ and the Gauss curvature $K$ is nonnegative. It follows from Remark 2.2 that

$$
\frac{1}{2} \Delta S=P+(2-S) S \geq(2-S) S \geq 0 .
$$

So either $S=0$ and the surface is a geodesic sphere, or $S=2$ and the surface is the Clifford torus.

If the normal bundle is nowhere flat, we have that the Gauss curvature $K$ is positive from $K^{N} \leq 2 K$. It follows from $K+K^{N}=1$ in Theorem 3.3 that the assumption $K^{N} \leq 2 K$ is equivalent to $S \leq 4 / 3$. By Theorem 3.1 we get $S=4 / 3$ and $M$ is the Veronese surface in $S^{4}$. 
Remark 3.7. Theorem 3.6 generalizes Theorem 1.1 obtained by Baker and Nguyen [1] to arbitrary codimension for the surface with flat normal bundle or nowhere flat normal bundle.

Theorem 3.8. Let $M$ be a closed surface minimally immersed in $S^{n}$ with positive Gauss curvature satisfying $2 K \leq K^{N} \leq 5 K$, then either

(1) $K^{N}=2 K$ and it is the Veronese surface in $S^{4}$, or

(2) $K^{N}=5 K$ and it is the generalized Veronese surface in $S^{6}$.

Proof. It follows from $2 K \leq K^{N} \leq 5 K$ and the Gauss curvature $K$ is positive that the normal curvature is positive everywhere. It follows from $K+K^{N}=1$ in Theorem 3.3 that $2 K \leq K^{N} \leq 5 K$ is equivalent to $4 / 3 \leq S \leq 5 / 3$. By Theorem 3.2 we get $S=4 / 3$ and $M$ is the Veronese surface in $S^{4}$, or $S=5 / 3$ and $M$ is the generalized Veronese surface in $S^{6}$.

Next we will give some new results concerning the normal curvature.

Theorem 3.9. Let $M$ be a closed minimal surface immersed in $S^{n}$ with positive Gauss curvature and flat or nowhere flat normal bundle. If $0 \leq K^{N} \leq 2 / 3$, then either

(1) $K^{N}=0$ and it is a geodesic sphere, or

(2) $K^{N}=2 / 3$ and it is the Veronese surface in $S^{4}$.

Proof. Since the Gauss curvature $K$ is positive and the normal bundle is flat or nowhere flat, it follows from Theorem 3.3 that $K+K^{N}=1$. So $0 \leq K^{N} \leq 2 / 3$ is equivalent to $0 \leq S \leq 4 / 3$. By Theorem 3.1 we get $S=0$ and $M$ is a geodesic sphere, or $S=4 / 3$ and $M$ is the Veronese surface in $S^{4}$.

Theorem 3.10. Let $M$ be a closed minimal surface immersed in $S^{n}$ with positive Gauss curvature. If $2 / 3 \leq K^{N} \leq 5 / 6$, then

(1) $K^{N}=2 / 3$ and $M$ is the Veronese surface in $S^{4}$; or

(2) $K^{N}=5 / 6$ and $M$ is a generalized Veronese surface in $S^{6}$.

Proof. We observe that the condition $2 / 3 \leq K^{N} \leq 5 / 6$ implies that the normal bundle is nowhere flat. It follows from Theorem 3.3 that $K+K^{N}=1$. So $0 \leq$ $K^{N} \leq 2 / 3$ is equivalent to $4 / 3 \leq S \leq 5 / 3$. By Theorem 3.1 we get $S=4 / 3$ and $M$ is the Veronese surface in $S^{4}$, or $S=5 / 3$ and $M$ is the generalized Veronese surface in $S^{6}$.

We conclude this paper with the following theorem.

Theorem 3.11. Let $M$ be a closed minimal surface immersed in $S^{n}$ with positive Gauss curvature. If $K^{N}$ is non-zero constant everywhere on $M$, then $K$ is constant and the immersion is one of the generalized Veronese surfaces.

\section{ACKNOWLEDGMENTS}

The author was partially supported by Chern Institute of Mathematics. The authors would like to thank the referees for their professional suggestions about this paper which led to various improvements. 


\section{REFERENCES}

1. C. Baker, H. T. Nguyen, Codimension two surfaces pinched by normal curvature evolving by mean curvature flow, Ann. I. H. Poincaré-AN.(2017) 34, 1599-1610.

2. K. Benko, M. Kothe, K. D. Semmler, U. Simon, Eigenvalues of the laplacian and curvature, Colloq. Math. (1979) 42, 19-31.

3. E. Calabi, Minimal immersions of surfaces in Euclidean spheres, J. Diff. Geom. (1967) 1, 111-125.

4. S. S. Chern, On the minimal immersions of the two-sphere in a space of constant curvature, Problems in analysis (Lectures at the Sympos. in honor of Salomon Bochner, Princeton Univ., Princeton, N.J., 1969), pp. 27-40. Princeton Univ. Press, Princeton, N.J., 1970.

5. B. Y. Chen, Classification of Wintgen ideal surfaces in Euclidean 4-space with equal Gauss and normal curvatures, Ann. Glob. Anal. Geom. (2010) 38, 145-160.

6. M. do Carmo, N. Wallach, Representations of compact groups and minimal immersions into spheres, J. Diff. Geom. (1970) 4, 91-104.

7. J. Q. Ge, Z. Z. Tang, A proof of the DDVV conjecture and its equality case, Pacific J. Math. (2008) 237, 87-95.

8. Z. H. Hou, D. Yang, Minimal Surfaces in a Unit Sphere, Journal of Mathematical Research with Applications (2012) 32, 346-354.

9. K. Kenmotsu, On compact minimal surfaces with non-negative gaussian curvature in a space of constant curvature: I, Tohoku Math. J. (1973) 25, 469-479.

10. K. Kenmotsu, On compact minimal surfaces with non-negative gaussian curvature in a space of constant curvature: II, Tohoku Math. J. (1975) 27, 291-301.

11. M. Kozlowski, U. Simon, Minimal immersion of 2-manifolds into spheres, Math. Z. (1984) $186,377-382$.

12. H. B. Lawson, Local rigidity theorems for minimal hypersurfaces, Ann. Math. (2) (1969) 89, 187-197.

13. Z. Lu, Normal scalar curvature conjecture and its applications, J. Funct. Anal. (2011) 261, 1284-1308.

14. C. K. Peng, Z. Z. Tang, On surfaces immersed in Euclidean space $R^{4}$, Science China Mathematics (2010) 53(1), 251-256.

15. U. Simon, Eigenvalues of the Laplacian and minimal immersions into sphere, In: Differntial Geometry (L.A. Cordero, ed.), Pitman, Montreal, 1985, pp.115-120.

School of Mathematics, Liaoning University, Shenyang, China

E-mail address: dlutyangdan@126.com 